Accelerator Department

BROOKHAVEN NATIONAL LABORATORY

Associated Universities, Inc.

Upton, New York 11973

AGS DIVISION TECHNICAL NOTE

№. 145

\title{
GAUSS CLOCK CALIBRATION
}

H. Weisberg

March 13, 1978

The "gauss clock" consists of a voltage to frequency converter driven by the voltage picked up on a winding on the backleg of the E20 magnet. The gauss clock counts provides a number that is related to the main magnet field and therefore the proton momentum. The nominal calibration was set up to be

$$
P(\mathrm{MeV} / \mathrm{c})=0.5 \mathrm{G}
$$

where $G$ is the gauss clock up-counts minus down-counts starting from reference time $T_{0}$.

If the gauss clock is linear, it can be calibrated by measuring the frequency of the accelerating $\mathrm{rf}$ and the beam radius for various gauss clock readings during the acceleration cycle. Define the following quantities:

$$
\begin{aligned}
& \mathrm{m}=\text { proton mass }=938.2796 \mathrm{MeV} / \mathrm{c}^{2} \\
& c=\text { velocity of light }=2.99792458 \times 10^{10} \mathrm{~cm} / \mathrm{sec} \\
& r=\text { average radial displacement of the beam with respect } \\
& \text { to the horizontal pick-up electrodes } \\
& r_{0}=\text { radius of that equilibrium orbit for which } r=0 \\
& f=r f \text { frequency } \\
& f_{0}=\text { frequency for a particle with velocity } c \text { on the equi- } \\
& \text { librium orbit of radius } r_{0} \\
& \mathrm{~h}=\text { harmonic number of the AGS }=12 \\
& \bar{\gamma}_{t r}=\text { transition gamma } \\
& \mathrm{P}_{\mathrm{o}}=\text { momentum of a proton that is on the equilibrium orbit } \\
& \text { of radius } r_{0} \text {. }
\end{aligned}
$$


Then the rf frequency is given by

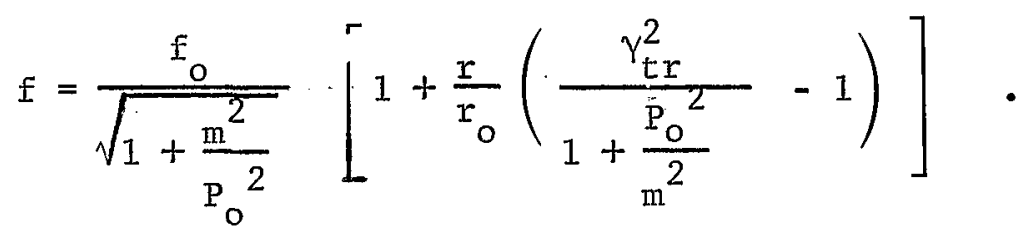

Now let us assume that the integrated gauss clock counts are linearly related to the quilibrium orbit momentum

$$
P_{0}=G_{1}\left(G+G_{0}\right)
$$

Here $G_{1}$ and $G_{0}$ are calibration constants to be determined; $G_{1}$ should be approximately $0.5 \mathrm{MeV} / \mathrm{c}$ per count and $G_{0}$ is related to the field at time $T_{0^{*}}$ Since $r_{0}$ is related to $f_{0}$ by

$$
r_{0}=\frac{h c}{2 \pi f_{0}}
$$

we can rewrite Eq. (1) as

$$
f=\frac{f_{o}}{\sqrt{1+\left[\frac{m}{G_{1}\left(G+G_{0}\right)}\right]^{2}}}\left\{1+\frac{2 \pi f_{0}}{h c}\left[\frac{\gamma_{t r}^{2}}{1+\left[\frac{G_{1}\left(G+G_{0}\right)^{2}}{m}-1\right.}\right] \cdot(4)\right.
$$

A set of measurements of $f, r$ and $G$ at 27 different times during the acceleration cycle was made. The frequency $f$ was measured with a Hewlett Packard 5345A $500 \mathrm{MHz}$ time base digital frequency ratio counter. A gate time of 100 usec was used for momenta up to $4.5 \mathrm{GeV} / \mathrm{c}$. From 4.5 to $9 \mathrm{GeV} / \mathrm{c}$, where greater frequency accuracy was needed and the frequency was changing less rapidly, a $1 \mathrm{msec}$ gate was used. Above $9 \mathrm{GeV} / \mathrm{c}$ a $10 \mathrm{msec}$ gate was used. The gauss clock reading was always taken at a time later than the frequency meter "arm" pulse by an amount equal to one half the gate time, so that the effect of the frequency rise with time cancelled to first order.

The mean radius $r$ was obtained from the average of the signals from the 46 pick-up electrodes that were available at the time of the measurement. 
The frequency measurements were fit to Eq. (4) by non-linear least squares, treating $f_{0}, G_{1}$ and $G_{0}$ as free parameters. To avoid magnet saturation effects, only data below $15 \mathrm{GeV} / \mathrm{c}$ were used. A constant value $\gamma_{\text {tr }}^{2} \approx$ $v_{H}^{2} \approx 8.7^{2}$ was used. An uncertainty of $\pm 0.05 \mathrm{~cm}$ was assumed for each radius measurement. The results ${ }^{*}$ were

$$
\begin{aligned}
& f_{0}=(4.457165 \pm 0.000015) \times 10^{6} \mathrm{~Hz} \\
& G_{1}=0.50568 \pm 0.00016 \mathrm{MeV} / \mathrm{c} \text { per count } \\
& G_{0}=107.0 \pm 0.6 \text { counts } .
\end{aligned}
$$

By Eq. (3), the measured value of $f_{0}$ gives $r_{0}=5057.43$ in., which agrees with the AGS nominal design radius of $5057.4 \mathrm{in}$.

We now have two different measurements of momentum: from the gauss clock by the equation

$$
P=G_{1}\left(G+G_{0}\right)\left(1+\frac{r}{r_{0}} \psi_{t r}^{2}\right)
$$

and from the rf frequency by

$$
P=\beta \mathrm{m} / \sqrt{1-\beta^{2}} \text { where } \beta=\left(f / f_{0}\right)\left(1+r / r_{0}\right) \text {. }
$$

Figure 1 gives the ratio of the momenta determined by these two methods for the various points measured. The fact that this ratio is equal to one to within a few tenths of a per cent below $15 \mathrm{GeV} / \mathrm{c}$ is a measure of the internal consistency of the measurement. Above $15 \mathrm{GeV} / \mathrm{c}$, a systematic discrepancy sets in, reaching $1.5 \%$ at $28 \mathrm{GeV} / \mathrm{c}$. Such an effect is expected from magnet saturation.

From the measurements we can work backward and apply a correction (including the magnet saturation effect) to quoted values of AGS momentum for recent operation:

$$
\text { Nominal Momentưum }
$$

$28.2 \mathrm{GeV} / \mathrm{c}$

28.2
Corrected: Momentum.

$$
28.9 \mathrm{GeV} / \mathrm{c}
$$$$
29.0
$$

(Fast Extracted Beam)

(S1ow Extracted Beam)

\footnotetext{
*The long term stability of the gauss clock has not been investigated. The quoted uncertainties correspond only to the internal consistency of the measurements at one time.
} 
The FEB value is for a radius at extraction of $r=-0.9 \mathrm{~cm}$, which corresponds to recent operating conditions. The SEB value is for $r=+0.2 \mathrm{~cm}$, which corresponds to the radius at which $v=8-2 / 3$ for recent settings of the ring quadrupoles.

HW/ 1sk

TO: Dept. Admin. AD Physicists AGS Div. Engineers

MCR

E. Gi11

R. Warkentien

T. Wild 
DATA OF 3 P $7>3$

RATIO OF NONENTUM FROM R.F. FREQUENCY TO MOMENTUP FRDM GAUSS CLOCK $F Q=4457.16540 \mathrm{KHZ} ; \mathrm{G} 1=0.50568$ MEU $/ \mathrm{C}$ PER COUHT; GG= 107.0 COUNTS; ERRDR BARS CORRESFOND TO AN UNCERTAINTY IN RADIUS DF G. GSO CH.

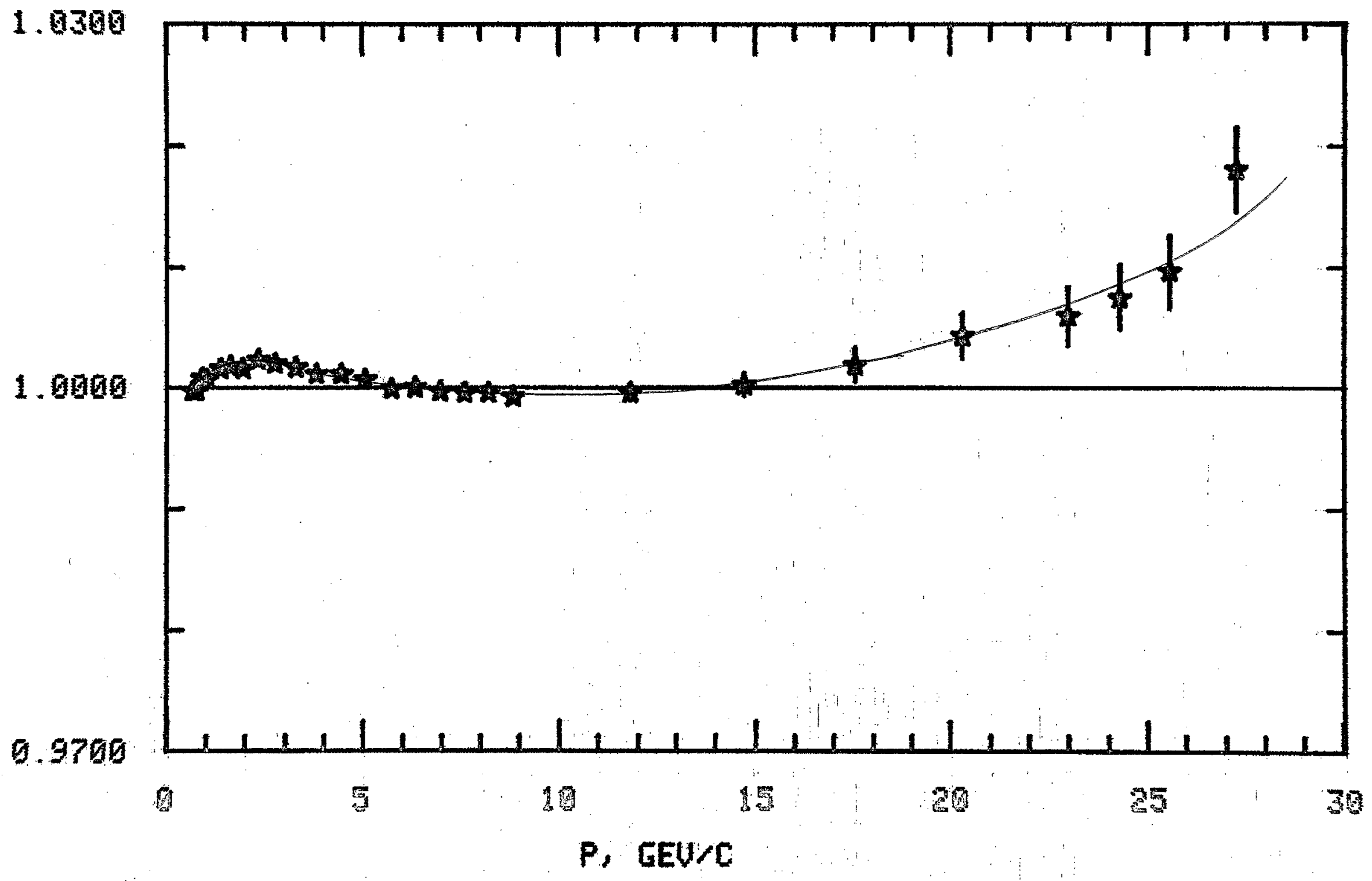

Fig. 1 